Voix et Images

voixetimages

\title{
Bibliographie Philippe Haeck (1969-1980)
}

\section{Philippe Haeck}

Volume 6, numéro 3, printemps 1981

Philippe Haeck

URI : https://id.erudit.org/iderudit/200279ar

DOI : https://doi.org/10.7202/200279ar

Aller au sommaire du numéro

Éditeur(s)

Les Presses de l'Université du Québec

ISSN

0318-9201 (imprimé)

1705-933X (numérique)

Découvrir la revue

Citer ce document

Haeck, P. (1981). Bibliographie Philippe Haeck (1969-1980). Voix et Images, 6(3), 373-380. https://doi.org/10.7202/200279ar d'utilisation que vous pouvez consulter en ligne.

https://apropos.erudit.org/fr/usagers/politique-dutilisation/ 


\section{Bibliographie Philippe Haeck (1969-1980)}

Note 1. Tout y est sauf: les textes qu'on a publiés sans m'en envoyer de copie; les textes parlés de quelques interviews ou entretiens avec Yves Boisvert, Paul-André Bourque, Michel Gay, Jacques Godbout, Wilfrid Lemoine, Jacques Marchand; un texte intitulé «Considérations sur le professeur* paru en novembre 1965 dans /Engagé fle journal des étudiants de l'École normale Ville-Marie) dont je ne trouve plus de copie.

Note 2. Comme j'attache beaucoup d'importance aux titres j'ai rétabli le titre de dix-neuf articles parus au Devoir; j'ai indiqué mon titre entre crochets et précédé d'un astérisque à la suite du titre inscrit dans le journal (le responsable de ces altérations était le responsable du cahier).

Note 3. J'ai suivi l'ordre chronologique: j'aime voir comment une histoire, ici la mienne, demeure fidèle à certaines lignes de force, rompt ou renoue avec d'autres. $J$ 'ai mis pêle-mêle toutes les formes de texte allant de la simple information au livre - à chaque fois il s'agit de tenter d'écrire. Quand plusieurs titres sont répertoriés sous le mème numéro, it s'agit soit d'informations ou de poèmes parus dans une même publication qui n'ont pas été regroupées sous un titre commun.

Ph. H.

\section{9}

1. «Chronologie de la vie et de l'ceuvre de Paul-Marie Lapointe". La Barre du jour. nos 17-18-19-20, janvier-août 1969, pp. 282-284.

2. "Le Vierge incendié de Paul-Marie Lapointe», Ibid., pp. 285-297.

1970

3. «Histoire de la littérature française. Berthelot Brunet ». Prospectives, vol. 6, no 5 , novembre 1970, p. 350.

\section{2}

4. «Contre l'audio-visuel». Tagore. Revue d'information pédagogique du Collège de Maisonneuve, vol. 1, no 2, janvier 1972, pp. 5-8.

5. «Presqu'Amérique et la littérature dans les cégep». Tagore, vol. 1, no 3, février 1972, pp. 4-5.

6. «Roland Barthes ou le cours comme parole paisible". Tagore, vol. 1, no 4, mars 1972, pp. 12-13. 
7. Le Vierge incendié, une nouvelle écriture. Mémoire de maîtrise, Université de Montréal, septembre 1972, $97 \mathrm{p}$.

8. «La pédagogie après mai 1968». Tagore, vol. 2, no 2, octobre 1972, pp. 1-12.

9. «L'enseignement de I'histoire littéraire». Tagore, vol. 2, no 3, novembre 1972. pp. 8-12.

\section{3}

10. «La pédagogie après mai 1968 : persienne du troisième volet $\%$. Tagore, vol. 2, no 5 , janvier 1973, pp. 29-31.

11. «Naissance de la poésie moderne au Québec". Études françaises, vol, IX, no 2 , mai 1973, pp. 95-113.

12. «pour la révolution», xpour paul-marie lapointe». Les Herbes rouges, no 9 , juin 1973, s. p., pp. 9-12.

13. "car tendresse jamais n'abîmera le vrai: lecture de Irish Coffees au no name bar \& Vin rouge valley of the moon». Hobo Québec, nos 9-10-11, octobrenovembre 1973, p. 7.

14. «Le rôle de l'enseignement de la littérature dans la formation générale de l'étudiant au cégep». Le Trait d'union. Journal des étudiants du C.É.G.E.P. de Maisonneuve, vol. 34, no 5, 13 novembre 1973, p. 7.

15. «Rien à dire de la tuile...*. Textes. Revue du département de français du Collège de Maisonneuve,, no 1, décembre 1973, p. 13.

16. «Arthur Rimbaud, l'écriture dangereusen. Ibid., pp. 64-74.

1974

17. «Suite logique». Hobo Québec, nos 14-15, janvier 1974, p. 16.

18. «Les herbes rouges polysèment», Ibid., p. 23.

19. «Pour un enseignement raisonné de la littérature». Libre cours. Revue d'action pédagogique des enseignants du Collège de Maisonneuve, vol. 1, no 1, janvier 1974, pp. 10-14.

20. «La «sagesse» amérindienne». Hobo Québec, nos 16-17, février-mars 1974, pp. 40-41.

21. Nattes. Les Herbes rouges, no 18, mars 1974, s.p., 36 p.

22. ««poésie canadienne» 1963-1973». Hobo Québec, no 18, avril-mai 1974, pp. 2527.

23. Traduction de «Etudiant ou nègre" (1967) de Jerry Farber. Libre cours, vol. 1, no 4, mai 1974, pp. 47-54.

24. «la jeune femme», «il ne sert à rien». Les Herbes rouges, no 21, juin 1974, s. p., p. 16.

25. «Ecriture et enseignement». lbid., pp. 37-38.

26. «Le soleil, l'œil». Préface à Genèses de Paul Chamberland, Montréal, l'Aurore, 1974, pp. 11-13.

27. «Philippe Haeck». Petit Catalogue d'activités culturelles 74-75, Atelier d'expression multidisciplinaire, automne 1974, p. 22. 
1975

28. "Quel amour". Chroniques, no 1, janvier 1975, pp. 44-48.

29. "Le Clitoris de la fée des étoiles», «Poèmes du vent et des ombres». Ibid., p. 81.

30. «Pour un enseignement réaliste de la littérature». Textes, no 5, janvier 1975 , pp. 14-33.

31. L'Action restreinte. De la littérature. «Écrire», Montréal, l'Aurore, 1er trimestre $1975,112 \mathrm{p}$.

32. «La folie, la tristesse». Chroniques, no 2, février 1975, pp. 37-40.

33. "L'art d'être grand-père», «Le créateur de Rrose Sélavy». lbid., pp. 66, 68.

34. Tout va bien. «Lecture en vélocipède», Montréal, I'Aurore, 1er trimestre 1975. $96 \mathrm{p}$.

35. «La poésie en 1974». Chroniques, no 3, mars 1975, pp. 42-45.

36. "Hélène Cixous». Ibid., p. 78.

37. "D'André Roy à Madeleine Gagnon* ["Deux écritures modernes]. Le Devoir, 22 mars 1975, p. 15.

38. "Le nouveau Paul-Marie Lapointe: du neuf et du moins neuf» [*Des poèmes d'amour]. Le Devoir, 29 mars 1975, p. 14.

39. «La gêne respiratoire», «Troublée». Cross Country: Special Issue: Montréal, nos 3-4, 1975, pp. 21-22.

40. "La tête me brûle». Chroniques, no 4, avril 1975, pp. 34-38.

41. "Quand Chamberland et Garneau se font un cadeau ». ["Les dieux et la politique]. Le Devoir, 12 avril 1975, p. 14.

42. «Deux livres petits et denses *. [ Pour la dissémination]. Le Devoir, 19 avril 1975, p. 14.

43. «Des contes pour qui? ». Chroniques, no 5, mai 1975, pp. 30-34.

44. "La bande dessinée québécoise», Ibid., p. 78.

45. "A propos de l'avenir de l'évaluation ». Libre cours, vol. 2, no 4, mai 1975, pp. 25-26.

46. «Les conditions de l'écriture au Québec». Textes, no 6, mai 1975, pp. 42-49.

47. «Un travail de mélancolique, signé Jacques Brault * [ Le travail de la mélancolie]. Le Devoir, 3 mai 1975, p. 17.

48. «Être au service d'une cause: honorable mais difficile [ ["Poésie et politique]. Le Devoir, 10 mai 1975, p. 24.

49. «Corps d'oiseaux: notes sur la poésie d'Anne Hébert « [ 'Le corps des oiseaux]. Le Devoir, 17 mai 1975, p. 25.

50. «Lacroix/Lapointe/Charlebois: trois recueils traditionnels» [ Une poésie traditionnelle ]. Le Devoir, 31 mai 1975, p. 17.

51. «Les conditions de l'écriture au Québec». Chroniques, nos 6-7, juin-juillet 1975 , pp. 3-11.

52. «Les yeux complémentaires". lbid., pp. 75-84.

53. «La question du style». Ibid., pp. 127-130.

54. "La critique sociale de Desbiens et Toupin [ [ Modernité et mythologies]. Le Devoir. 7 juin 1975, p. 17. 
55. «Brossard et Massé, ou deux écritures désirantes * [ Deux écritures désirantes]. Le Devoir, 21 juin 1975, p. 13.

56. «Quand les poètes veulent parler de mort* [ "Les poètes et la mort]. Le Devoir, 5 juillet 1975, p. 12.

57. «Les étudiants, la poésie». Chroniques, nos 8-9, août-septembre 1975, pp. $132-136$.

58. «Bulletin de bibliographie «, "Pour la critique», «En lutte!». Ibid., pp. 155-158.

59. «Roger Des Roches et les «corps fantômes»" ["Corps fantômes]. Le Devoir, 2 août 1975, p. 12.

60. «Encore (et toujours) l'amour » ["Encore l'amour]. Le Devoir, 16 août 1975, p. 12.

61. «Ce que sera la saison prochaine [ ["La prochaine saison de poésie]. Le Devoir, 6 septembre 1975, p. 14.

62. «Prendre les armes et prendre la parole " ["La prise de la parole]. Le Devoir, 27 septembre 1975, p. 15.

63. $\propto$ Des cours intéressants». Chroniques, no 10, octobre 1975, pp. 43-46.

64. «Une nouvelle revue littéraire», „Prochaines parutions à Parti Pris». Ibid., pp. 74, 75.

65. «Parler québécois et écrire québécois». Chroniques, no 11, novembre 1975. pp. 14-16.

66. «Les mots et les masses». Le Devoir, 8 novembre 1975, p. 14.

67. «La collection «Paroles»?». Chroniques, no 12, décembre 1975, pp. 37-40.

68. «Hélène Cixous», «Lecture en vélocipède». lbid., pp. 77, 79.

1976

69. *Pour une écriture montante». Chroniques, no 13, janvier 1976, pp. 63-68.

70. «De Garneau à Gervais* ["De Michel Garneau à André Gervais]. Le Devoir, 3 janvier 1976, p. 9.

71. «Du surréalisme au mysticisme» [ La femme et l'enfant]. Le Devoir, 17 janvier 1976, p. 14.

72. «John Ashberry ou le raisonnement * [ John Ashberry ou les nattes du raisonnement ]. Le Devoir, 31 janvier 1976, p. 17.

73. «Désir : de classe, d'individu ». Chroniques, no 14, février 1976, pp. 20-22.

74. aL'urgence de la critique». Ibid., pp. 62-67.

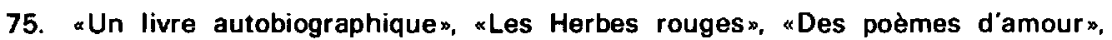
«L'avant-garde $\nsim, ~ « Q u ' e s t-c e$ que la dialectique? », «Le divan du pauvre et les vieux docteurs», «Les murs de l'école», «La force de l'oubli». Ibid., pp. 87-94.

76. «La marque de Barthes $\%$. Le Devoir, 21 février 1976, p. 16.

77. «La poésie en 1975». Chroniques, no 15, mars 1976, pp. 39-52.

78. «Parler le corps $»$. Ibid., pp. 94-95.

79. «Apollinaire, le dernier poète». Le Devoir, 13 mars 1976, p. 22.

80. «A travers le Québec». Chroniques, no 16, avril 1976, p. 10.

81. «Une veillée au corps $\bowtie$. Ibid., pp. 38-39. 
82. "Qui dit mieux ?», "Nous taire?". Ibid., pp. 91-92, 94-95.

83. «Des catastrophes intimes». Le Devoir, 24 avril 1976, p. 15.

84. «Poésie et griefs». Le Devoir, 1er mai 1976, p. 14.

85. «Pour la création». Chroniques, no 17, mai 1976, pp. 34-47.

86. «Exposition », «L'air me manque $n$, «Science et politique $», \propto B d$ et révolutionnaires . lbid., pp. 89-92.

87. «La contre-culture ou la culture révolutionnaire». Chroniques, nos 18-19, juin-juillet 1976, pp. 11-14.

88. "Philippe Haeck questionne Paul Villeneuve, écrivain». Ibid., pp. 134-158.

89. «Me vient un nouveau discours». Ibid., pp. 159-175.

90. «Éditions Parti Pris». Jbid., pp. 190-191.

91. "Des fleurs de bonne volonte". Le Devoir, 5 juin 1976, p. 15.

92. "Mallarmé, la chute de l'auteur". Le Devoir, 26 juin 1976, p. 13.

93. "Femmes», "A la clarté», "La ligne bleue». Cheval d'attaque (Paris), nos 16-17: «Poésie au Québec. Une année de lectures», 1976, pp. 71-74.

94. «Une nouvelle revue de poésie», Le Devoir, 3 juillet 1976, p. 12.

95. «Contre l'ignorance». Le Devoir, 10 juillet 1976, p. 12.

96. «Difficile poésie». Le Devoir, 17 juillet 1976, p. 15.

97. «Conflit». Chroniques, nos 20-21, août-septembre 1976, pp. 31-45.

98. «Pamphlétaires et étudiants», «Les contes québécois». ibid., pp. 189-192.

99. «Engorgements poétiques». Le Devoir, 14 août 1976, p. 13.

100. "La leçon de Descartes». Le Devoir, 21 août 1976, p. 12.

101. «Toutes les fées mal tournées». Le Devoir, 11 septembre 1976, p. 12.

102. "Le Rire de la Reine (notes sur les Sables du rêve de Thérèse Renaud)" (en collaboration avec Claire Savary). Voix et images, vol. II, no 1, septembre 1976, pp. 13-19.

103. Les dents volent. Les Herbes rouges, nos 39-40, septembre 1976, 52 p.

104. «Texte lu à la nuit des écritures intervenantes en juillet 1976». Dérives, nos 5-6, 1976, pp. 37-40.

105. "La théorie critique de l'école de Francfort», «Gilgamesh», «Chacun pour soix. Chroniques, no 22, octobre 1976, pp. 93-94.

106. "Lire la poésie formaliste». Le Devoir, 2 octobre 1976, p. 22.

107. *Marteau et plumeau ». Le Devoir, 18 décembre 1976, p. 16.

108. «La force des femmes». Le Devoir, 31 décembre 1976, p. 12.

1977

109. «Ancienne et nouvelle poésie». Le Devoir, 5 février 1977, p. 18.

110. «Dire des merveilles». Le Devoir, 26 février 1977, pp. 17-18.

111. «Le messianisme québécois ou Ceci est une fiction*. Chroniques, no 27, mars 1977, pp. 36-63. 
112. «Pour l'action ». Change (Paris, Seghers/Laffont), nos 30-31 : «Souverain Québec», mars 1977, pp. 146-151.

113. "L'oreille heureuse dans les herbes rouges". Le Devoir, 19 mars 1977, p. 18.

114. Nattes. Cistre. Cahiers trimestriels des lettres différentes (Lausanne, L'Âge d’Homme), no 1 : «Québec (presque) libre», 1er trimestre 1977, pp. 117-126.

115. "Le soleil dans les nuages», «S'étirer nu», *Écoute bien». Canton s'met à faire de la poésie, les Presses étudiantes, Sherbrooke, septembre 1977, pp. 73-76.

116. "Note sur le malaise de l'enseignement». Prospectives, vol. 13, no 3, octobre 1977, p. 134.

117. Adequation des services de la bibliothèque aux pratiques pédagogiques du cégep (en collaboration avec Louise Beauregard, Luce Goulet-Côté et Yves Dupont). Recherche subventionnée par la D.G.E.C., Collège de Maisonneuve, novembre $1977,99 \mathrm{p}$.

118. $\alpha$ Je parle ce qui m'agite». Chroniques, nos 29-30-31-32, automne 1977 hiver 1978, pp. 216-220.

119. "Le déjeuner des canotiers". Ibid., pp. 312-313.

1978

120. "La poésie québécoise aujourd'hui». Magazine littéraire (Paris), no 134, mars 1978, pp. 79-83.

121. Polyphonie. Roman d'apprentissage. VLB éditeur, 1er trimestre 1978, 312 p.

122. "Le hasard et l'attention». La Nouvelle Barre du jour, no 71, novembre 1978, pp. 24-51.

123. "Notes premières sur la ville». Dérives, nos 14-15, 1978, pp. 53-58.

124. Naissances. De l'écriture québécoise. Thèse de doctorat, Université de Sherbrooke, novembre 1978, $524 \mathrm{p}$.

125. «Des cours d'écriture dans une séquence création au collège de Maisonneuve». Québec français, no 32, décembre 1978, pp. 48-49.

126. "Le travail de création». Québec français, supplément au no 32: «le français au collégial», décembre 1978, pp. 40-42.

1979

127. "Une petite voix». Possible, vol. 3, no 2, hiver 1979, p. 120

128. Polyphonie. Roman d'apprentissage. Deuxième édition, VLB éditeur, 2e trimestre

128. Polyphonie. Roman d'apprentissage. Deuxième édition, VLB éditeur, 2e trimestre 1979, $312 \mathrm{p}$.

129. «Perdre son corps. Une méthodologie pour l’étude du «corps romanesque»: une lecture de I'Amélanchier». Présence francophone, no 18, printemps 1979, pp. 127-133.

130. Naissances. De l'écriture québécoise. VLB éditeur, 2e trimestre 1979, $410 \mathrm{p}$.

131. "Philippe Haeck». Petit dictionnaire des écrivains, Union des écrivains québécois, $2 e$ trimestre 1979 , p. 83. 
132. «Philippe Haeck a lu Lueur de Madeleine Gagnon». La P'tite Presse littéraire. Magazine d'information de VLB éditeur, mai 1979, p. 2.

133. «Mélançon: une lecture agitée» ["Une lecture agitée]. Le Devoir, 23 juin 1979. p. 17.

134. «Tremblement et certitude». Spirale, no 1, septembre 1979, p. 7.

135. «Lire la bible». Ibid., p. 10.

136. «La souffrance infinie». Ibid., p. 11.

137. «Partir du plus simple». La Nouvelle Barre du jour, no 81, septembre. 1979, pp. 5-24.

138. «Poéthique des Herbes rouges». Dérives, no 19, 3e trimestre 1979, pp. 39-53.

139. «La séparation». Spirale, no 3, novembre 1979, pp. 4-5.

140. «Furieusement jeune». Ibid., p. 7.

141. «Pour Saint-Denys Garneau ». La Nouvelle Barre du jour, no 83, octobre, novembre 1979, pp. 60-70.

142. "La carte de visite volée». Livres et auteurs québécois 1978, les Presses de l'Université Laval, 4e trimestre 1979, pp. 202-203.

143. "La parole verte». Le Devoir, 24 novembre 1979, p. XVII.

144. «Godbout Pirouette angoisse»[ décembre 1979, p. 9.

1980

145. "La marche». Spirale, no 5 , janvier 1980, p. 5.

146. «Jubilation: une rencontre avec Kenneth White». Ibid., p. 11.

147. *Blessures*. Spirale, no 6, février 1980, pp. 15 et 22.

148. «Une femme écarlate». Courrier du centre international d'études poétiques (Bruxelles), nos 135-136: «Autour de Claire Lejeune», janvier-mars 1980 , pp. 41-45.

149. «Les policiers, vedettes de l"histoire». Spirale, no 8, avril 1980, p. 13.

150. «De la curiosité». La Nouvelle Barre du jour, nos 90-91, mai 1980, pp. 71-98.

151. «Le Cahier d'amour (extrait)». Ibid., pp. 99-100.

152. «De l'amnésie *. Ibid., pp. 221-222.

153. «Let my children hear music $»$. Spirale, no 10, juin 1980, pp. 12, 15.

154. «Ce matin», «Mélisande crie», «Sa chevelure bouclée $»$ « Six heures , «L'air frais de maix. Mensue/ 25. Journal d'urgence tiré à blanc (Herstal, Belgique, Atelier de l'agneau), no 42, juin 1980, s.p.

155. "La parole verte». La Nouvelle Barre du jour, no 92-93, juin 1980, pp. 151-158.

156. "L'apprentissage de l'écriture». Livres et auteurs québécois 1979. les Presses de I'Université Laval, 4ème trimestre 1980, pp. 167-170. 


\section{Principales lectures}

Joseph Bonenfant, «Polyphonie. D'une écriture ouvrière». La Nouvelle Barre du jour, no 71, novembre 1978, pp. 79-83.

François Charron, «Comment ça s'écrit». Chroniques, no 2, février 1975, pp. 8-11.

Normand de Bellefeuille, «Haeck; la révolution à la portée de la main». La Presse, 18 décembre 1976, p. C4.

Jean Fisette, «L'écriture moderne. Encore. Enfin». Voix et images, vol. IV, no 1, septembre 1978, pp. 148-150.

André Gervais, «Pour l'appeau éthique. De la juxtaposition ", La Nouvelle Barre du jour, no 96 , novembre 1980, pp. 69-76.

Réjean Jacques, «Chanson et écriture $*$ La Presse, 15 mars 1975, p. D6.

Réjean Jacques, «Tout va bien». La Presse, 22 novembre .1975, p. D4.

Wladimir Krysinski, «Polyphonie. Roman d'apprentissage». Livres et auteurs québécois 1978, Presses de l'Université Laval, 1979, pp. 123-126.

Laurent Laplante, «Littérature et pédagogie». Le Soleil, 24 septembre 1979, p.

Robert Mélançon, «Philippe Haeck, poète révolutionnairex. Le Devoir, 3 juin 1978, p. 27.

Robert Mélançon, «Haeck: des essais discutables». Le Devoir, 9 juin 1979, p. 17 et 16 juin 1979 , p. 17.

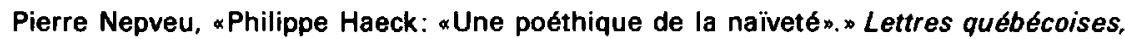
no 13, février 1979, pp. 22-24.

Jacques Pelletier, aL'enseignement de la littérature aujourd'hui». Liberté, no 103 , janvier-février 1976, pp. 79-86.

Jean Royer, «Philippe Haeck: l'écriture comme un travail du désir d'inventer et de changer la société». Le Soleil. 26 avril 1975, p. D9.

Max Roy, «Philippe Haeck. Tout va bien». Livres et auteurs québécois 1975, Presses de I'Université Laval, 1976, pp. 114-115.

Max Roy, «Polyphonie. Roman d'apprentissage». Livres et auteurs québécois 1978 , Presses de I'Université Laval, 1979, pp. 126-129. 